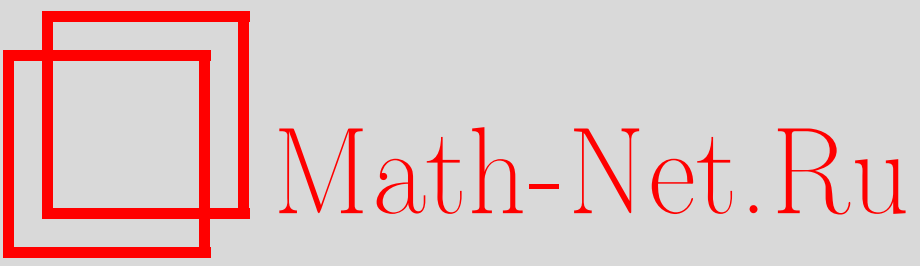

С. Н. Лакаев, М. Х. Шерматов, О спектре гамильтониана одной системы трех квантовых частиц на решетке, УМH, 1999, том 54, выпуск 6, 165-166

DOI: https://doi.org/10.4213/rm240

Использование Общероссийского математического портала Math-Net.Ru подразумевает, что вы прочитали и согласны с пользовательским соглашением

http://www . mathnet.ru/rus/agreement

Параметры загрузки:

IP: 52.23 .180 .231

26 апреля 2023 г., 13:43:49 


\title{
О СПЕКТРЕ ГАМИЛЬТОНИАНА ОДНОЙ СИСТЕМЫ ТРЕХ КВАНТОВЫХ ЧАСТИЦ НА РЕШЕТКЕ
}

\author{
С.Н. ЛАкаев, М.Х. ШеРматов
}

Одним из замечательных результатов спектрального анализа трехчастичного оператора Шрёдингера на евклидовом пространстве является эффект Ефимова, т.е. существование бесконечного числа трехчастичных связанных состояний, энергии которых накапливаются к нижнему краю существенного спектра (см. [1]-[3]). Аналогичный эффект имеет место для гамилтониана трех произвольных и трех одинаковых частиц на решетке (см. [4], [5]), более того, наличие этого явления существенно зависит от значений полного квазиимпульса [5].

В настоящем сообщении для гамилтониана одной системы трех квантовых частиц (два фермиона взаимодействуют с третьей частицей другой природы) получено компактное интегральное уравнение Фаддеева-Ньютона для собственных функций, описан существенньй спектр и установлено существование эффекта Ефимова в случае, когда масса фермиона в достаточной степени больше массы третьей частицы. Кроме того, этот результат в отличие от резултата работы [5] основан на изучении спектра предельного фаддеевского оператора в подпространстве функций с угловым моментом $l=1$. Отметим, что установленный нами результат вполне согласуется с результатом о наличии эффекта Томаса для аналогичной системы трех частиц с точечными взаимодействиями.

Пусть $T^{3}$ - трехмерный тор, $L_{2}\left(\left(T^{3}\right)^{3}\right)$ - гильбертово пространство квадратично интегрируемых функций, определенных на $\left(T^{3}\right)^{3}$, а $L_{2}^{a}\left(\left(T^{3}\right)^{3}\right)$ - подпространство пространства $L_{2}\left(\left(T^{3}\right)^{3}\right)$, состоящее из функций $f\left(k_{1}, k_{2}, k_{3}\right)$, антисимметрических относительно переменных $k_{1}$ и $k_{2}$.

В импульсном представлении гамильтониан данной системы действует в пространстве $L_{2}^{a}\left(\left(T^{3}\right)^{3}\right)$ по формуле:

$$
\begin{aligned}
& \left(H_{\mu, \lambda} f\right)\left(k_{1}, k_{2}, k_{3}\right)=\left(\varepsilon\left(k_{1}\right)+\varepsilon\left(k_{2}\right)+\lambda \varepsilon\left(k_{3}\right)\right) f\left(k_{1}, k_{2}, k_{3}\right) \\
& \quad-\mu \sum_{\alpha=1,2} \int_{\left(T^{3}\right)^{3}} \delta\left(k_{\alpha}-k_{\alpha}^{\prime}\right) \delta\left(k_{\beta}+k_{3}-k_{\beta}^{\prime}-k_{3}^{\prime}\right) f\left(k_{1}^{\prime}, k_{2}^{\prime}, k_{3}^{\prime}\right) d k_{1}^{\prime} d k_{2}^{\prime} d k_{3}^{\prime},
\end{aligned}
$$

$\alpha \neq \beta, \alpha, \beta=1,2$. Здесь $\delta(k)$ - трехмерная дельта-функция Дирака, $\varepsilon(k)$ - вешественно аналитическая функция в некоторой комплексной окрестности тора $T^{3}$, удовлетворяющая условию $\varepsilon(k)=\varepsilon(-k)$ для любого $k \in T^{3}, \lambda>0$ - отношение масс фермиона и 3 -й частицы, $\mu>0-$ энергия взаимодействия.

Пусть $K=k_{1}+k_{2}+k_{3}$ - квазиимпульс системы, а $F_{K}=\left\{\left(k_{1}, k_{2}, k_{3}\right) \in T^{3}: k_{1}+k_{2}+k_{3}=K\right\}$ - шестимерное многообразие. Обозначим через $L_{2}^{a}\left(F_{K}\right)$ гильбертово пространство квадратично интегрируемых функций, определенных на $F_{K}$ и удовлетворяющих условию:

$$
f\left(k_{1}, k_{2}, k_{3}\right)=f\left(k_{1}, k_{2}, K-k_{1}-k_{2}\right)=-f\left(k_{2}, k_{1}, K-k_{1}-k_{2}\right)=-f\left(k_{2}, k_{1}, k_{3}\right) .
$$

Пространство $L_{2}^{a}\left(\left(T^{3}\right)^{3}\right)$ и оператор $H_{\mu, \lambda}$ представляются в виде следующих прямых интегралов (см. [5]):

$$
L_{2}^{a}\left(\left(T^{3}\right)^{3}\right)=\int_{T^{3}} \oplus L_{2}^{a}\left(F_{k}\right) d K, H_{\mu, \lambda}=\int_{T^{3}} \oplus \widehat{H}_{\mu, \lambda}(K) d K
$$

где ограниченный самосопряженный оператор $\widehat{H}_{\mu, \lambda}(K), K \in T^{3}$, действует в пространстве $L_{2}^{a}\left(F_{K}\right)$ по формуле:

$$
\begin{array}{r}
\widehat{H}_{\mu, \lambda}(K) f(p, q, K-p-q)=\varepsilon_{K, \lambda}(p, q) f(p, q, K-p-q) \\
-\mu \int_{T^{3}}\left[f\left(p, q^{\prime}, K-p-q^{\prime}\right)+f\left(q^{\prime}, q, K-q-q^{\prime}\right)\right] d q^{\prime} .
\end{array}
$$

Здесь $\varepsilon_{K, \lambda}(p, q)=\varepsilon(p)+\varepsilon(q)+\lambda \varepsilon(K-p-q), K \in T^{3}, 0<\lambda<\infty$.

Предположим, что функция $\varepsilon(p)$ имеет единственный невырожденный минимум в точке $p_{0} \in T^{3}$ 
Лемма. Пусть $K_{0}=3 p_{0} \in T^{3}$. Тогда существует аналитическая функция $P_{\lambda}(K)$, определенная в некоторой $\beta_{\lambda}$-окрестности $U_{\beta_{\lambda}}\left(K_{0}\right)$ точки $K_{0}$, такая, что для любого $K \in U_{\beta_{\lambda}}\left(K_{0}\right)$ точка $\left(P_{\lambda}(K), P_{\lambda}(K)\right)$ является единственной точкой минимума функиии $\varepsilon_{K, \lambda}(p, q)$.

Пусть $\widehat{L}(K)$ - подпространство функций $f(p, q)$ из $L_{2}\left(\left(T^{3}\right)^{2}\right)$, удовлетворяющих условию $f(p, q)=-f(q, p)$, а $H_{\mu, \lambda}(K), K \in T^{3},-$ ограниченный самосопряженный оператор, действующий в $\widehat{L}(K)$ по формуле:

$$
\left(H_{\mu, \lambda}(K) f\right)(p, q)=\varepsilon_{K, \lambda}(p, q) f(p, q)-\mu \int_{T^{3}}\left[f\left(p, q^{\prime}\right)-f\left(q, q^{\prime}\right)\right] d q^{\prime} .
$$

Для любого $K \in T^{3}$ оператор $H_{\mu, \lambda}(K)$ унитарно эквивалентен оператору $\widehat{H}_{\mu, \lambda}(K)$, действующему в $L_{2}^{a}\left(F_{K}\right)$ по формуле (3).

Положим

$$
z(K, \lambda)=\min \varepsilon_{K, \lambda}(p, q), \quad \mu(K, \lambda)=\min \left(\int_{T^{3}} \frac{d q}{\varepsilon_{K, \lambda}(p, q)-z(K, \lambda)}\right)^{-1} .
$$

Теорема 1. Для любых $K \in T^{3}, \mu>0, \lambda>0$ оператор $H_{\mu, \lambda}(K)$ не имеет собственных значений $z>\max \varepsilon_{K, \lambda}(p, q)$.

Теорема 2. Пусть $K \in T^{3}$ u $\mu \neq \mu(K, \lambda)$. Тогда дискретный спектр оператора $H_{\mu, \lambda}(K)$ конечен.

Предположим, что для нокоторого $K \in U_{\beta_{\lambda}}\left(K_{0}\right)$ выполняется соотношение:

$$
\mu(K, \lambda)=\left(\int_{T^{3}} \frac{d q}{\varepsilon_{K, \lambda}\left(P_{\lambda}(K), q\right)-z(K, \lambda)}\right)^{-1} .
$$

Обозначим через $\lambda_{0}$ решение уравнения

$$
\frac{2(1+\lambda)^{2}}{\pi \lambda \sqrt{1+2 \lambda}}-\frac{(1+\lambda)^{2}}{\pi \lambda^{2}}\left(\pi-2 \arccos \frac{\lambda}{1+\lambda}\right)=1, \quad 0<\lambda<\infty .
$$

ТеОрема 3. Пусть $\lambda>\lambda_{0}, K \in U_{\beta_{\lambda}}\left(K_{0}\right)$ и в точке $K$ выполняется равенство (5). Тогда оператор $H_{\lambda}(K)=H_{\mu}(K, \lambda), \lambda(K)$ имеет бесконечное число собственных значений $E_{\lambda, 1}(K), E_{\lambda, 2}(K), \ldots, E_{\lambda, n}(K), \ldots$ таких, что $\lim _{n \rightarrow \infty} E_{\lambda, n}(K)=z(K, \lambda)$.

\section{СПИСОК ЛИТЕРАТУРЫ}

[1] Ефимов В.Н.// Ядерная физика. 1970. Т. 12. №5. С. 1080-1091. [2] Яфаев Д. К. // Матем. сб. 1974. Т. 9 (136). № 4 (8). С. 567-592. [3] Меркурьев С. П., Фаддеев Л. Д. Квантовая теория рассеяния для систем нескольких частиц. М.: Наука, 1985. [4] Лакаев С. Н. // ТМФ. 1991. Т. 89. № 1. С. 94-104. [5] Л акаев С. Н. // Функц. анализ и его прил. 1993. Т. 27. №3. C. $15-28$.

Принято редколлегией 02.10.1999 